\title{
A Metaheuristic Bandwidth Allocation Scheme for FiWi Networks Using Ant Colony Optimization
}

\author{
Panagiotis Sarigiannidis \\ and Malamati Louta \\ Dept. of Informatics \\ and Telecommunications Engineering \\ University of Western Macedonia \\ Kozani, Greece \\ Email: \{psarigiannidis,louta\}@uowm.gr \\ Ilenia Tinnirello \\ Degli Studi Di Palermo \\ University of Palermo \\ Palermo, Italy \\ Email: ilenia.tinnirello@tti.unipa.it
}

\author{
Georgios Papadimitriou \\ and Petros Nicopolitidis \\ Dept. of Informatics \\ Aristotle University of Thessaloniki \\ Thessaloniki, Greece \\ Email: \{gp,petros\}@csd.auth.gr
}

\author{
Konstantinos Diamantaras \\ Dept. of Information Technology \\ Alexander Technological Institute \\ of Thessaloniki Thessaloniki, \\ Greece \\ Email: kdiamant@it.teithe.gr
}

\author{
Christos Verikoukis \\ Centre Tecnologic de Telecomunicacions de Catalunya \\ Castelldefels \\ Barcelona, Spain \\ Email: cveri@cttc.es
}

\begin{abstract}
Optical-wireless access networks constitute a quite attractive solution to meet the ever-increasing bandwidth requirements of end-users, offering significant benefits such as ubiquitous coverage in the wireless domain and huge bandwidth in the optical domain. However, converging optical and wireless networking technologies, with Passive Optical Networks (PONs) and $4 \mathrm{G}$ wireless standards, such as the Worldwide Interoperability for Microwave Access (WiMAX) and the Long Term Evolution (LTE), entails major challenges that need to be addressed. In this context, designing an efficient and fair bandwidth distribution with Quality of Service (QoS) support is a difficult task due to the interdependence of resource allocation process to both optical and wireless domains. Specifically, the bandwidth distribution in the optical domain has to be aware of the mobile user heterogeneity in the wireless domain, while in the wireless domain the allocation process depends on the optical transmission grant opportunities. In this work a metaheuristic approach to govern the bandwidth allocation in modern Fiber Wireless (FiWi) networks is proposed. The Ant Colony Optimization (ACO) method is effectively employed in order to optimize the bandwidth report process of Mobile Subscribers (MSs). Simulation results reveal the effectiveness of the proposed approach in terms of latency and network throughput.
\end{abstract}

\section{INTRODUCTION}

Hybrid optical-wireless access networks, known also as Fiber-Wireless (FiWi) networks, leverage on the complementary advantages of both optical and wireless networking technologies, combining the capacity of optical networks with the mobility and ubiquity offered by the wireless networks. Thus, they are considered to provide a viable solution to support multi-application and bandwidth demanding services such as internet protocol television, video on demand and Voice Over Internet Protocol (VoIP). However, a number of research issues still need to be efficiently addressed in order to enable the smooth integration of both optical and wireless domains. Bandwidth distribution in FiWi networks is still in its infancy, while the consideration of fairness in the overall design is much less met in related research [1]. This work focuses on the bandwidth distribution process with the aim to provide a cognitive fair and efficient resource allocation using the Ant Colony Optimization (ACO) concept. By optimizing the bandwidth allocation process in FiWi networks, the performance of the data delivery is dramatically improved, allowing more users to be served, more application to be offered, and high standards of Quality of Service (QoS) to be addressed [2]. By utilizing an ACO framework that governs the polling process of Mobile Subscribers (MSs), a cognitive approach on determining the traffic requests of each MS is feasible. The ACO framework is utilized in order to determine the optimal polling result during the phase of bandwidth reporting. Each MS is polled, and the ACO decides the additional traffic request of this MS. Thus, the waiting time of the new traffic arrivals is reduced, since the coordinator grants more bandwidth that the reported bandwidth. Simulation results reveal the effectiveness of the so-called Ant Dynamic Bandwidth Allocation (AntDBA) scheme compared to a benchmark DBA scheme.

The remainder of this paper is organized as follows. Section II briefly presents the background of FiWi networks. In Section IV, the proposed FiWi DBA scheme is presented, while Section V presents the performance evaluation environment as well as obtained simulation results. Finally, Section VI concludes this paper.

\section{BACKGROUND}

Ethernet-based standards tend to efficiently integrate an optical-wireless network due to their common physical and design features such as the common Ethernet framing. In this 


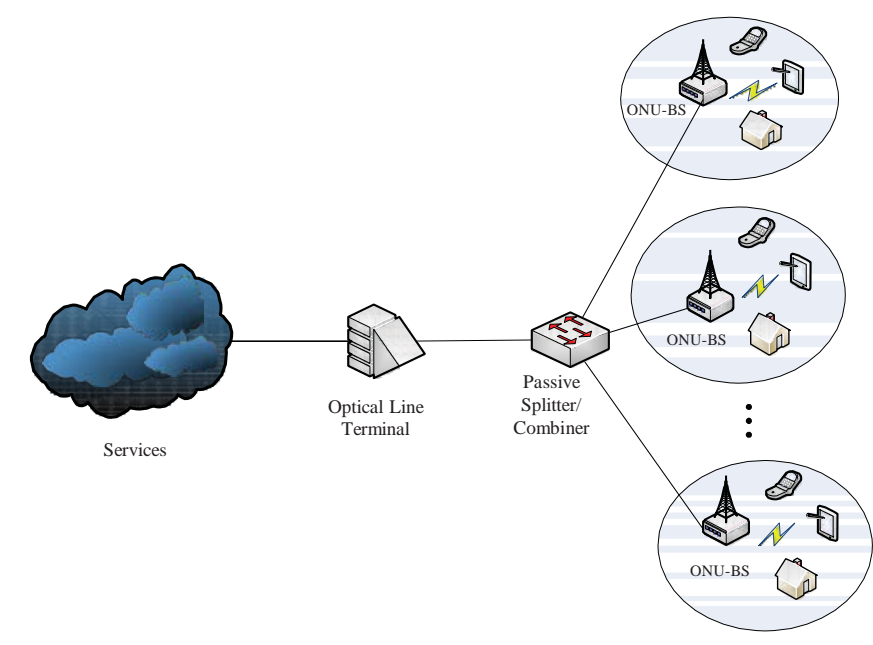

Fig. 1. The architecture of the proposed FiWi network. The optical backhaul is integrated with multiple wireless access networks in the front-end.

work, we consider a 10 Gbps Ethernet Passive Optical Network (10G-EPON) integrated with several Worldwide Interoperability for Microwave Access (WiMAX) front-end access Base Stations (BSs). This type of integration seems to gain the main attention since the underlying architecture presents several advantages, such as a) similarities in the bandwidth allocation process and b) common QoS provisioning. Figure 1 illustrates the architecture of the proposed FiWi network.

At the 10G-EPON domain, an Optical Line Terminal (OLT) that resides in the providers office is connected to a number of Optical Network Units (ONUs) near subscriber premises via a passive splitter/combiner. An integrated ONU-BS in a single device is used, being capable of deriving full information from bandwidth requests and allocations in both optical and wireless networks. ONU and BS are connected independently through a standard Ethernet interface. A software-based bridge is placed between the ONU and BS in order to manage the bandwidth distribution process and support the QoS between 10G-EPON and WiMAX systems.

Concerning the bandwidth allocation process, the MultiControl Protocol (MPCP) is utilized in the 10G-EPON domain between the OLT and the ONU-BSs. The MPCP involves the GATE message and the REPORT message which govern the bandwidth allocation operation. Through sending a GATE message that includes a transmission opportunity (defining transmission time and transmission duration) to each ONU$\mathrm{BS}$, the OLT assigns the transmission window to each ONUBSs. On the other hand, the REPORT message is utilized by each ONU-BS in order to inform the OLT about its queue occupancy and request transmission time for the next frame. In the WiMAX domain, a two way communication is realized, with the MSs sending data to the corresponding ONU-BS in the uplink direction and the BS delivering data to the connected MSs in a broadcast manner in the downlink direction. The Time Division Duplexing (TDD) technique is usually used, where each frame consists of two separate, successive sub-frames, one for each direction. WiMAX adopts Orthogonal Frequency Multiple Division Multiple Access (OFDMA), enabling multiple MSs to utilize different bandwidth regions in the time and frequency domains. Concerning QoS provisioning, 10G-EPON service classes are divided into three categories, namely Expedited Forwarding (EF), Assured Forwarding (AF) and Best Effort (BE). EF service class has the highest priority and includes services where delay and jitter are of outmost importance (e.g., voice). AF category involves bandwidth guaranteed applications (e.g., video on demand), while low priority applications and services with no strict requirements in delay or throughput are categorized in BE class (e.g., e-mail, web browsing). On the other side, the BS related packet classifier classifies uplink data into the five services of WiMAX, namely, UnSolicited Grant Services (UGS), real-time Polling Service (rtPS), extended real-time Polling Service (ertPS), non real-time Polling Service (nrtPS) and Best Effort (BE). Based on the aforementioned, it is obvious that a common QoS mapping policy has to be defined in the integrated FiWi network. In this work, the triple priority policy is adopted, where the three priority classes defined by the 10G-EPON constitute the main QoS differentiation services classes of the FiWi network. Therefore, the priorities of the WiMAX have to be accordingly adapted to the three priorities of the 10G-EPON. Specifically, since ertPS and rtPS are quite similar, both supporting real-time service flows, they could easily be treated in the same manner. Additionally, nrtPS and BE could be merged in one service class. Thus, the five WiMAX service categories are aggregated into three types, namely UGS, rtPS and BE.

\section{RELATED WORK}

Bandwidth allocation scheme proposals in FiWi networks are rarely found in related research literature. This may be attributed to the fact that the bandwidth allocation process in FiWi is a quite difficult task due to the interdependency presented in the optical and wireless domains.

In [3] a Hierarchical QoS-Aware DBA (HQA-DBA) algorithm is presented, applied in both OLT and ONU-BS. Bandwidth resources are assigned on the basis of user requests and priority queue weights. It is shown that the proposed scheme decreases average network delay and packet drop probability. In [4], a DBA algorithm is proposed for an integrated Wavelength Division Multiplexing PON (WDM-PON) with multi-channel wireless network, ensuring a minimum guaranteed bandwidth, while sharing the excess bandwidth amongst the connected wireless users. [5] introduces a DBA scheme, namely Slotted DBA (S-DBA), aiming at increasing the channel utilization by reducing the signaling overhead caused by cascaded bandwidth requests and grants. This is achieved by synchronizing the allocation timing of the granted opportunities. In [6], the authors introduce a QoSaware DBA scheme applied in a FiWi network composed of EPON and WiMAX networks. The proposed DBA algorithm, namely WiMAX EPON DBA (WE-DBA), consists of two separate DBA algorithms implemented in the OLT 
and ONU-BS respectively, offering however an integrated QoS provisioning solution assuming differentiated traffic service guarantees. WE-DBA is deemed as a benchmark DBA scheme; its architecture engages an EPON and several frontend WiMAX networks. WE-DBA consists of two separated DBA algorithms implemented in the OLT and the ONU-BS respectively. The algorithm handles the polling management in the optical domain, where the MPCP is adopted untouched. WE-DBA presents two main drawbacks, i.e., it entails a predefined fixed amount of bandwidth for BE traffic and it neglects the fairness issue. Another indicative example can be found in [4]. The proposed Frame-Based DBA (FB-DBA) scheme intends to reduce the end-to-end delay of the WiMAX traffic and to offer QoS provisioning across the EPON and WiMAX domains. A schedule algorithm was presented in [7] in order to reduce the transmission latency in the ONU with high utilization efficiency of bandwidth. The Inter-channel and Intra-channel Dynamic Wavelength/Bandwidth Allocation (IIDWBA) algorithm [8] introduced a schedule strategy based on the excess distribution policy. The game theory concept is utilized in [9] so as to ensure fair bandwidth allocation providing equal queuing delays to all wireless nodes. However it is limited only to mesh wireless systems.

\section{ANT-DBA}

\section{A. Metaheuristics}

Metaheuristics are efficient methods that might lead to optimization solutions to a variety of problems. In essence, they constitute computational methods that persistently endeavor to find an optimized solution, if any, to a generic problem that has a potential to be improved or resolved. They rely to experience-based techniques which are structured using history data. Normally, they compare current with past data and try to detect a better current solution than the current global solution in order to replace the global with the current solution. If an optimal solution exists, these techniques are capable of finding it after a set of continuous iterations or cycles. ACO is a metauristic technique and tries to detect an optimal solution with several trial and error attempts.

\section{B. ACO Technique}

The optimization potential of the ant colony concept was inspired by the observation of the ant life on handling the ant colonies. The ants present a bahavior that allows them to find the shortest path to food sources from their basis using chemical pheromone. An amount pheromone is is trailed on the ground; hence, ants can sense it and they tend to choose the path that is sensed most dense in pheromone. In essence, each ant trip from and back to a food source operates as an iteration. It updates the pheromone amount of this path and therefore leads other ants to this path with a probability. However, as the time passes the amount of pheromone, including all paths, is evaporated. Only the shortest path to the food sources tend to maintain or/and increase its pheromone levels since new ants select this path towards their target. After a set of continuous iterations, the ants that follow on searching food sources know the optimal path so far.

\section{Bandwidth Allocation Modeling}

The upstream bandwidth coordination is modeled and optimized in this work since the bandwidth management in the downstream direction is an uncomplicated task. We consider that the bandwidth allocation process is divided in bandwidth rounds. The OLT polls all ONU-BSs during each bandwidth round. In each $t$ bandwidth round each ONU-BS reports its bandwidth occupancy in order to get a bandwidth grant in the next $t+1$ bandwidth round. The FiWi network consists of $\mathrm{N}$ ONU-BSs. Assume that $\mathrm{R}_{\mathrm{i}}(\mathrm{t})$ denotes the report of the $i$ ONU-BS during the bandwidth round $t$ in terms of bytes. Similarly, $\mathrm{G}_{\mathrm{i}}(\mathrm{t})$ stands for the granted bandwidth to i ONUBS regarding the report during the previous round in terms of bytes. Hence, the granted bandwidth $\mathrm{G}_{i}(\mathrm{t})$ is associated with the report bandwidth $\mathrm{R}_{\mathrm{i}}(\mathrm{t}-1)$. Each ONU-BS reports bandwidth requests coming from the connected MSs. Assume that the uplink capacity of the wireless access BS is $\mathrm{C}_{\mathrm{w}}$ bytes. As a result, the threshold of a report message is $\mathrm{Cw}$, given that only MS users are considered in the network (no users exist in the optical interface of the ONU-BS). Hence it holds:

$$
\mathrm{R}_{\mathrm{i}} \leq \mathrm{C}_{W}
$$

$$
\mathrm{G}_{\mathrm{i}} \leq \mathrm{CW}_{\mathrm{W}}
$$

Due to transmission and propagation delays, new arrivals take place in each MS. Thus, during the $t$ bandwidth round an additional bandwidth amount of $\mathrm{N}_{\mathrm{i}}(\mathrm{t})$ bytes arrived in MSs that belong to $\mathrm{i}$ ONU-BS. However, the ONU-BS is granted $\mathrm{G}_{\mathrm{i}}(\mathrm{t})$ which corresponds to $\mathrm{R}_{\mathrm{i}}(\mathrm{t}-1)$ report. An optimal solution implies that the granted bandwidth to each ONU-BS would be equal to $\left.G_{i}(t)=R\right) i(t-1)+N_{i}(t)$. Nevertheless, the amount of $\mathrm{N}_{\mathrm{i}}(\mathrm{t})$ remains unknown and hard to be computed since it depends on several parameters such as a) the delay caused by the polling process and b) the MSs' arrival rate. Given that the traffic arrival estimation in access networks in a vague task due to the bursty traffic in the modern access networks, an alternative solution is needed.

\section{Problem Formulation}

In the light of the aforementioned remarks, we formulate the optimization bandwidth allocation problem as follows:

Given the $R_{i}(t-1)$ determine the amount of $G_{i}(t)$

$$
\text { so as to } \mathrm{G}_{\mathrm{i}}(\mathrm{t})=\mathrm{R}_{\mathrm{i}}(\mathrm{t}-1)+\mathrm{N}(\mathrm{t})
$$

\section{E. ACO Components}

We define the following $s$ trail states that correspond to possible values of $\mathrm{N}_{\mathrm{t}}$ :

$$
\mathrm{T}=\left\{\mathrm{T}_{1}, \mathrm{~T}_{2}, \ldots, \mathrm{T}_{\mathrm{s}}\right\}
$$


The first trail state corresponds to zero bandwidth, denoting that no additional bandwidth will be given. The last trail state denotes that the maximum additional bandwidth will be granted. In any case, the total granted bandwidth is not allowed to exceed the bandwidth threshold $\mathrm{Cw}$.

Each trail state is given an attractiveness factor, denoted by $\mathrm{F}_{\mathrm{ij}}(\mathrm{t})$, where $\mathrm{i}$ denotes the ONU-BS and $\mathrm{j}$ denotes the trail state. The attractiveness factor is expressed a a probability. Larger factor entails a more attractive trail. The initial values of the attractiveness factors are set as follows:

$$
\mathrm{F}_{\mathrm{j}}^{\mathrm{i}}(1)=\stackrel{1}{\mathrm{~s}^{\prime}}, \forall \mathrm{i}, \mathrm{j}
$$

A number of A ants is considered (for each ONU-BS). Each ant selects a possible trail state in a random way. Two ants are not allowed to select the same state; so a tabu search is applied. Each ant selects a trail during the bandwidth round $t$ and returns at the bandwidth round $t+1$. Assume that $S_{k}^{i}(t)$ denotes the selection of the $k$ ant during the $t$ bandwidth round, where $1 \leq \mathrm{k} \leq \mathrm{A}$. The attractiveness of the selected trail is assessed in the next bandwidth round $t+1$. If an ant selected the current optimal trail, then the attractiveness of the selected trails is increased. The current optimal trails is the actual additional amount of bandwidth that the $\mathrm{i}$ ONU-BS report at the following round, i.e., the $\mathrm{R}_{\mathrm{i}}(t+1)$.

Lastly, the evaporation process is modeled. At the end of each bandwidth round the pheromone of all trails is slightly evaporated by a factor $\rho$ :

$$
\mathrm{F}_{\mathrm{j}}^{\mathrm{i}}(\mathrm{t}+1) \leftarrow \mathrm{F}_{\mathrm{j}}^{\mathrm{i}}(\mathrm{t})-\rho \cdot \mathrm{F}_{\mathrm{j}}^{\mathrm{i}}(\mathrm{t}), \forall \mathrm{i}, \mathrm{j}
$$

The summation of all pheromone that was evaporated is calculated:

$$
\Delta^{\mathrm{i}}=\sum_{\substack{\mathrm{x}=1, \mathrm{x} /=\\ 0}}^{\lessgtr} \rho \cdot \mathrm{F}_{\mathrm{x}}^{\mathrm{i}}(\mathrm{t}) \forall \mathrm{i}
$$

For each bandwidth round where no current optimal found the $\Delta^{i}$ factor is increased by the levels of pheromone that were evaporated. On the contrary, the pheromone level of a trail found current optimal is increased by the $\Delta^{i}$ factor. If the current optimal corresponds to the o trail then:

$$
\mathrm{F}_{\mathrm{o}}^{\mathrm{i}}(\mathrm{t}+1) \leftarrow \mathrm{F}_{\mathrm{o}}^{\mathrm{i}}(\mathrm{t})+\Delta, \forall \mathrm{i}, \mathrm{j}
$$

As a next step the $\Delta^{i}$ factor is initialized:

$$
\Delta^{\mathrm{i}}=0, \forall \mathrm{i}
$$

\section{F. MS Fairness Allocation}

On the contrary to several DBA algorithms that assume a predefined $\mathrm{BE} / \mathrm{BE}$ bandwidth portion, such as [6], we propose a fair max-min allocation of the granted bandwidth $\mathrm{G}_{i}(\mathrm{t})$ to the connected MSs. First, the UGS/EF bandwidth requests are addressed using the max-min algorithm. Then, the rtPS/AF requests are accommodated using the surplus bandwidth. Finally, the BE/BE requests are processed.

\section{Performance Evaluation}

\section{A. Environment}

The Matlab simulation environment was used to numerically assess the performance of the proposed DBA scheme. A FiWi network was designed having an optical backhaul and several ONU-BSs at its front-end points. The optical part is governed by a 10G-EPON, while each wireless access front-end operates under the WiMAX standard. Hence, each ONU-BS is equipped with a double interface. In the optical edge, the ONU-BS follows the rules of an 10G-EPON compatible ONU. In the opposite side, it consists of a WiMAX BS. It is considered

that the two devices are directly connected; hence no additional process and waiting time is induced by bridging the two edges. The topology of the FiWi network is a tree topology.

The 10G-EPON uses a single channel with rate $1.25 \mathrm{Gbps}$ in the upstream direction. Another channel is utilized in the downstream direction with rate of 10.3125 Gbps. Each ONUBS is equipped with a logical buffer of 100 Mbytes. A guard time of 1 usec is inserted after each upstream allocation for avoiding collisions. The location of each ONU-BS was randomly selected assuming a RTT of $[200,400]$ usec.

The Partial Usage of Sub-Carriers (PUSC) technique is adopted in each WiMAX BS. In addition, the TDD technique is utilized. Hence, OFDMA method is applied to distribute time and frequency entities in each MS using a periodical frame. A downlink-to-uplink ratio of 1 : 1 was assumed yielding a frame of $10 \mathrm{~ms}$. A total number of 630 (resource) slots are available for allocating upstream bursts in the uplink sub-frame. According to the standard specifications, the BS is capable of utilizing four modulation schemes based on the MSs signal quality. Given that the bits per slot ratio depends on the underlying modulation scheme, a setting of 95 bits per slot at average was assumed. Thus, the wireless uplink capacity was equal to $\mathrm{C}_{\mathrm{w}}=7560$ bytes. As a result, each ONU-BS was permitted to report up to $\mathrm{Cw}_{\mathrm{w}}=7560$ bytes per

bandwidth cycle.

Regarding the MS traffic generation, we applied the following formula according to [6] assumptions. All MSs follow the same traffic profile. In particular, each MS generates three types of traffic classes, namely Constant Bit Rate (CBR) traffic (e.g., VoIP) of the UGS/EF priority class with average rate $1024 \mathrm{Kbps}$, Variable Bit Rate (VBR) traffic (e.g., streaming video) of the rtPS/AF priority class with average rate of $1 \mathrm{Mbps}$, and VBR traffic (e.g., file transfer) of the BE/BE priority class with average rate of $512 \mathrm{Kbps}$. The VBR traffic is generated in accordance with a self-similar probability distribution. More specifically, the burst size of each data stream was modeled by a Pareto distribution. Both shape parameters (idle and active) of the Pareto distribution was set equal to 1.5 .

Ant-DBA is assessed compared to WE-DBA [6] in three different versions having three different number of ants. The first version engages 10 ants, the second 20 and the third 30 ants. A total number of 101 trail states were considered, where the first state corresponds to 0 additional bandwidth, the second 


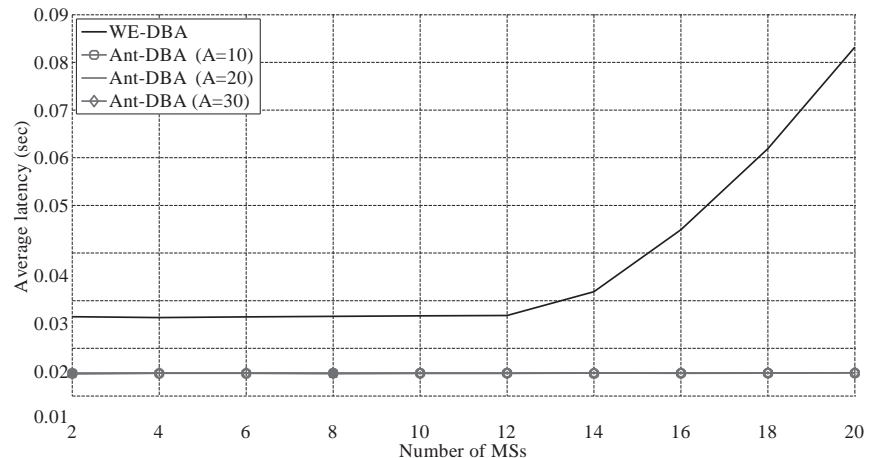

Fig. 2. Average data packet latency as the number of MSs increases. The number of ONU-BSs is 16.

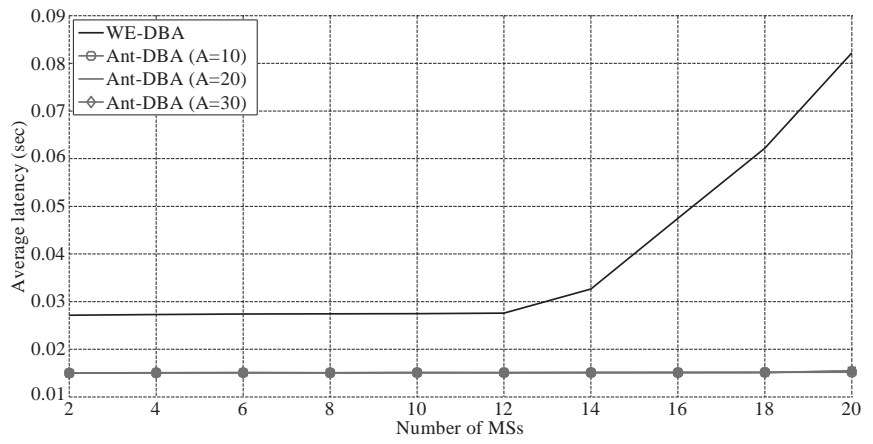

Fig. 3. Average data packet latency as the number of MSs increases. The number of ONU-BSs is 32 .

trail corresponds to $\frac{1}{100} \cdot \mathrm{CW}_{\mathrm{W}}$, the third trails to $\frac{2}{100} \cdot \mathrm{C}_{\mathrm{W}}$ and so on. The evaporation factor was set 0.01 . As mentioned before,

WE-DBA considers a fixed portion of bandwidth destined for accommodating $\mathrm{BE} / \mathrm{BE}$ traffic requests. This amount was set equal to 300 Kbps. The following numerical experiments reveal the performance of the two schemes in terms of latency and throughput.

\section{B. Results}

In the first set of experiments the average latency is investi-

gated. Figure 2 and Figure 3 demonstrate the average latency of the uplink direction in the FiWi network as the number

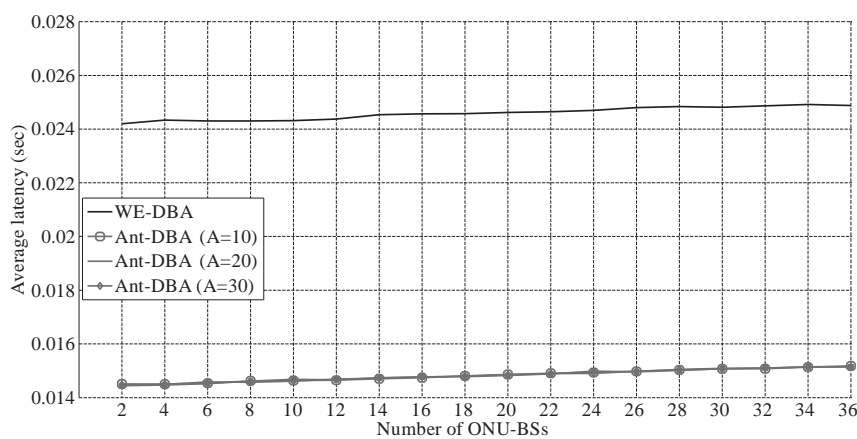

Fig. 4. Average data packet latency as the number of ONU-BSs increases. The number of MSs per ONU-BS is 10.

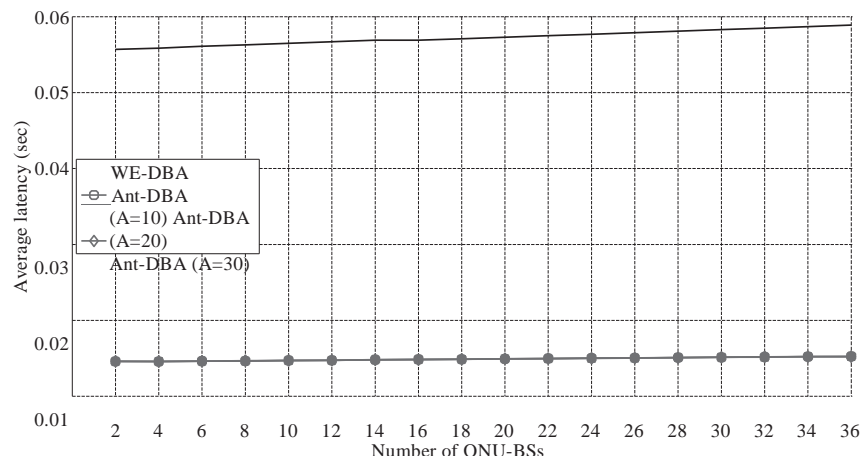

Fig. 5. Average data packet latency as the number of ONU-BSs increases. The number of MSs per ONU-BS is 20.

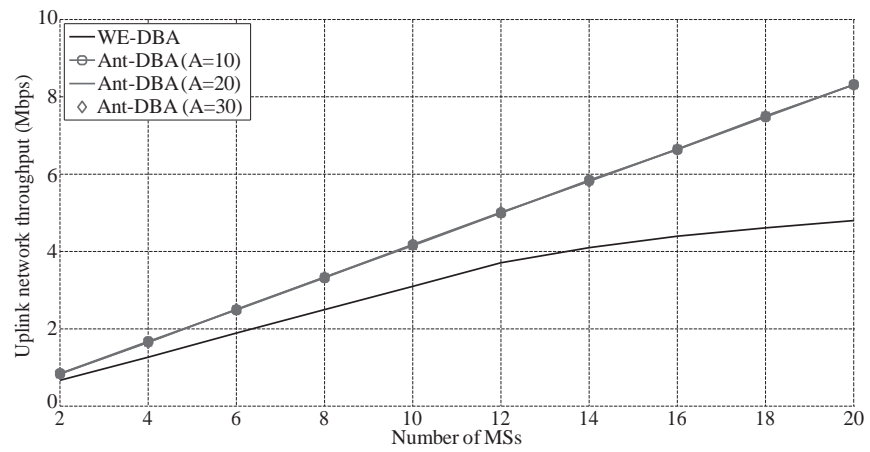

Fig. 6. Uplink network throughput as the number of MSs increases. The number of ONU-BSs is 16.

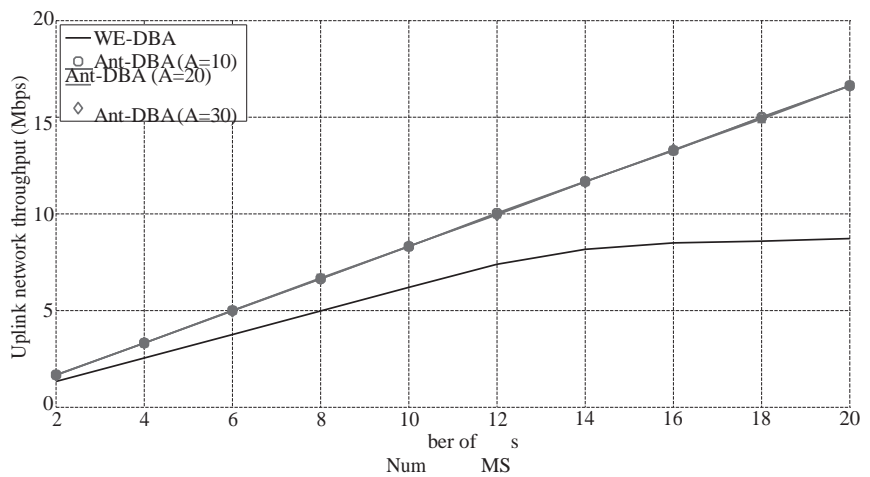

Fig. 7. Uplink network throughput as the number of MSs increases. The number of ONU-BSs is 32.

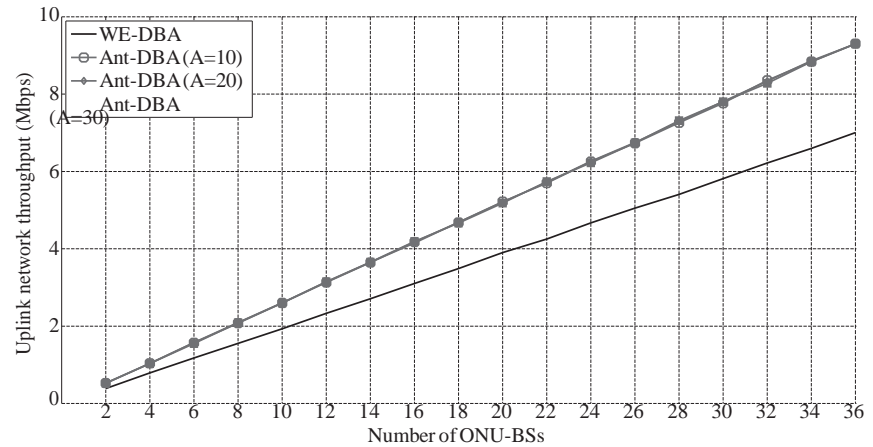

Fig. 8. Uplink network throughput as the number of ONU-BSs increases. The number of MSs per ONU-BS is 10. 


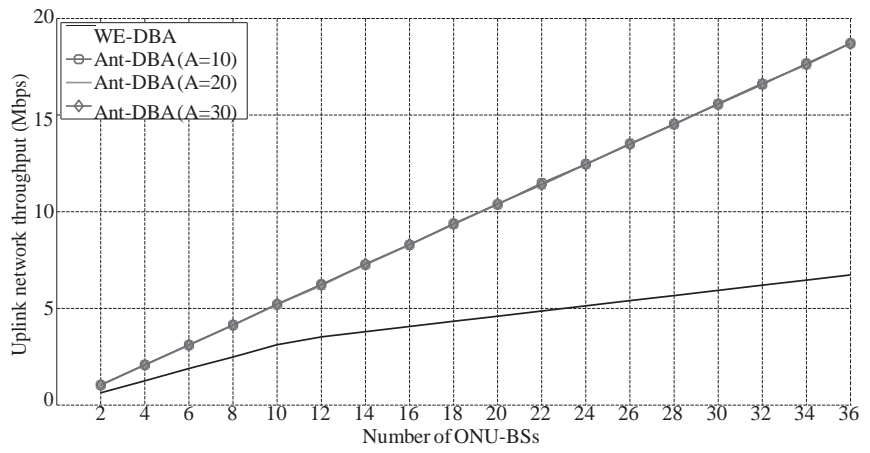

Fig. 9. Uplink network throughput as the number of ONU-BSs increases. The number of MSs per ONU-BS is 20.

of MSs per ONU-BS increases from 2 to 20. The number of ONU-BSs in the FiWi network remains unchanged and equal to 16. It is easy to observe that as the MS population becomes more dense, WE-DBA fails to provide an efficient bandwidth allocation among MSs. It seems that WE-DBA reaches a saturation point when the number of MSs is 12 . On the other hand, the proposed scheme presents a stable latency for all MS population points. It is clear that even before the saturation point Ant-DBA is constantly better than WEDBA since the ACO operation effectively improves the polling process by estimation the additional bandwidth each MSs need. On the contrary, the bandwidth redistribution of WEDBA seems problematic. This is happens due to the fact that it does not include any re-distribution process when allocating $\mathrm{BE} / \mathrm{BE}$ traffic.

Figure 4 and Figure 5 depict the average latency of the uplink direction in the FiWi network as the number of MSs per ONU-BS increases from 2 to 20 and the number of ONU-BSs is 32. The performance of Ant-DBA is quite better than that of WE-DBA, since it applies a more efficient MS bandwidth allocation. The underlying max-min technique permits the utilization of all network resources to the MSs without wasting bandwidth to requests that are granted more than they need.

The second set of figures explore the network throughput impact. Figure 6 and Figure 7 present the uplink network throughput in terms of Mbps when the number of MSs in the FiWi network is 10 and 20 respectively. As expected the performance of Ant-DBA excels the performance of WE-DBA. All versions of the proposed metaheuristic scheme achieve better performance than WE-DBA. Figure 8 and Figure 9 present the same results when the number of MSs is 20. Once more, the saturation point that WE-DBA experiences is reached when the number of MSs exceeds 12. In addition, WE-DBA causes a notable packet drop after the saturation point; hence the observed uplink throughput in that case collapses. On the other hand, Ant-DBA seems to ensure an efficient and high-throughput bandwidth management, since the observed packet delivery rate is increased as the number of MSs increases in a linear way.

\section{CONCLUSION}

ACO method was utilized in this work in order to optimize the polling method in modern FiWi networks. The bandwidth report of the connected MSs is enhanced to include the additional arrivals during the polling process. The proposed method sounds promising since it manages good performance in terms of latency and network throughput.

\section{ACKNOWLEDGMENT}

This research has been co-financed by the European Union (European Social Fund - ESF) and Greek national funds through the Operational Program "Education and Lifelong Learning" of the National Strategic Reference Framework (NSRF) - Research Funding Program: ARCHIMEDES III. Investing in knowledge society through the ESF. Also, it is supported by the AGAUR (2014SGR1551).

\section{REFERENCES}

[1] A. Sarigiannidis, M. Iloridou, P. Nicopolitidis, G. Papadimitriou, F. Pavlidou, P. Sarigiannidis, M. Louta, and V. Vitsas, "Architectures and bandwidth allocation schemes for hybrid wireless-optical networks," Communications Surveys Tutorials, IEEE, vol. 17, no. 1, pp. 427-468, Firstquarter 2015

[2] Z. Ghassemlooy, S. Arnon, M. Uysal, Z. Xu, and J. Cheng, "Emerging optical wireless communications-advances and challenges,” Selected Areas in Communications, IEEE Journal on, vol. 33, no. 9, pp. 1738-1749, Sept 2015.

[3] L. Jiang, M. lei Fu, and Z. chun Le, "Hierarchical qos-aware dynamic bandwidth allocation algorithm for wireless optical broadband access network," in Electronics, Communications and Control (ICECC), 2011 International Conference on, Sept 2011, pp. 4329-4332.

[4] C.-L. Lai, H.-T. Lin, H.-H. Chiang, and Y.-C. Huang, "Design and analysis of a frame-based dynamic bandwidth allocation scheme for fiber-wireless broadband access networks,” Optical Communications and Networking, IEEE/OSA Journal of, vol. 6, no. 5, pp. 486-500, May 2014.

[5] S. Ou, K. Yang, and H.-H. Chen, "Integrated dynamic bandwidth allocation in converged passive optical networks and ieee 802.16 networks," Systems Journal, IEEE, vol. 4, no. 4, pp. 467-476, Dec 2010.

[6] K. Yang, S. Ou, K. Guild, and H.-H. Chen, "Convergence of ethernet pon and ieee 802.16 broadband access networks and its qos-aware dynamic bandwidth allocation scheme," Selected Areas in Communications, IEEE Journal on, vol. 27, no. 2, pp. 101-116, February 2009.

[7] Z. Md Fadlullah, H. Nishiyama, Y. Kawamoto, H. Ujikawa, K.-I. Suzuki, and N. Yoshimoto, "Cooperative qos control scheme based on scheduling information in fiwi access network," Emerging Topics in Computing, IEEE Transactions on, vol. 1, no. 2, pp. 375-383, Dec 2013.

[8] N. Moradpoor, G. Parr, S. McClean, and B. Scotney, " $\{$ IIDWBA $\}$ algorithm for integrated hybrid $\{\mathrm{PON}\}$ with wireless technologies for next generation broadband access networks," Optical Switching and Networking, vol. 10, no. 4, pp. 439 - 457, 2013. [Online]. Available: http://www.sciencedirect.com/science/article/pii/S1573427713000544

[9] J. Coimbra, G. Schtz, and N. Correia, "A gamebased algorithm for fair bandwidth allocation in fibrewireless access networks," Optical Switching and Networking, vol. 10, no. 2, pp. $149-162$, 2013. [Online]. Available: http://www.sciencedirect.com/science/article/pii/S1573427712000756 